\title{
Performance of P2x7 and P2Y Purinergic Receptors as an Inhibiting Factor in the Progression of Pulmonary Neoplastic Cells
}

\author{
Matheus Ribeiro Bizuti ${ }^{1 *}$, Laura Nyland Jostl ${ }^{2}$, Emanuely Scramim ${ }^{1}$, \\ Keroli Eloiza Tessaro da Silva ${ }^{1}$, Luana Paula Schio ${ }^{1}$, \\ Gabriela Vidotto Cavallieri ${ }^{3}$, Renata Calciolari Rossi ${ }^{4}$ \\ and Débora Tavares de Resende e Silva ${ }^{5}$
${ }^{1}$ Federal University of Fronteira Sul, Chapecó, SC, Brazil.
${ }^{2}$ Federal University of Fronteira Sul, Passo Fundo, RS, Brazil.
${ }^{3}$ Environment and Regional Development at the University of Oeste Paulista, Presidente Prudente, $S P$, Brazil.
${ }^{4}$ University of Oeste Paulista, Presidente Prudente, SP, Brazil. \\ ${ }^{5}$ Program in Biomedical Sciences, Federal University of Fronteira Sul, Chapecó, SC, Brazil.
}

\section{Authors' contributions}

This work was carried out in collaboration among all authors. Authors MRB, LNJ and DTRS prepared the study design, in the literature review and participated in the writing of the manuscript. Authors ES,

KETS, LPS, GVC and RCS contributed to the literature review and the writing of the manuscript. All authors read and approved the final manuscript.

Article Information

DOI: $10.9734 / A R R B / 2020 / v 35 i 1230311$

Editor(s):

(1) Paola Angelini, University of Perugia, Italy.

Reviewers:

(1) Stefan Vasile Stefanescu, University of Chester, England. (2) B. Archana, Raja Rajeswari Medical College and Hospital, India. Complete Peer review History: http://www.sdiarticle4.com/review-history/63142

Mini-review Article

Received 12 September 2020 Accepted 23 November 2020 Published 11 December 2020

\section{ABSTRACT}

Different pathological processes are considered in lung cancer, such as nicotine deposition, oxidative stress, deregulation of metal ions and chronic inflammation. Purine signaling is involved in all processes, suggesting the importance of nucleotide receptors (P2X7 and P2Y) and adenosine receptors present in lung cells. The accumulation of toxic substances promotes chronic inflammation and cellular alteration, which induces the release of Adenosine Triphosphate (ATP) in 
the extracellular space, thus stimulating P2X7 receptors. The activation of P2X7 promotes an increase in the synthesis and release of some pro-inflammatory mediators. P2Y works by promoting the increase of intracellular calcium levels and, P2X7, changing cell permeability. Thus, drugs can be developed with a specific targeting for such recipients, thus provid additional therapeutic options to those existing.

Keywords: Adenosine triphosphate; cell proliferation; lung neoplasms; tobacco use disorder; purinergic signaling.

\section{INTRODUCTION}

Lung cancer is the leading cause of cancer death worldwide, with non-small cell lung cancer accounting for $85 \%$ of cases, and small cell lung cancer, $15 \%$ [1]. Lung cancer is more prevalent in men. The main risk factor associated with the cause of lung cancer is smoking since cigarette smoke has several carcinogenic components, among which the most evident are nicotine, tar and carbon monoxide. Thus, the most effective method to reduce the incidence of this neoplasia is to inhibit the use of tobacco [2].

The inflammatory responses developed by individuals affected by lung cancer contribute significantly to the progression of the disease. This is due to the potential capacity of inflammatory mediators to promote the proliferation of mutant cells [3]. These inflammatory mediators act both in decreasing and increasing cell proliferation. The type of response depends on the conditions of the environment [3]. Also during the inflammatory process, extracellular ATP contributes to the development of hyperalgesia, due to the activation of the $\mathrm{P} 2 \mathrm{X} 7$ receptor. Thus, this receptor is involved in the pain regulation process, as it is responsible for mediating sensitivity through the regulation of the nervous system [4].

In a chronic inflammatory condition, oxidative stress is present when reactive oxygen species (ROS) extrapolate their endogenous antioxidant capacity [5]. Thus, ROS, together with free radicals, trigger cell death by inducing apoptosis [5]. Neoplastic cells, as well as cells with mutative potential, are induced to death and, hence, the inflammatory condition is reduced. The P2X7 purinergic receptor acts in the positive regulation of inflammatory cells, therefore, the inhibition of this receptor would be a potential target for the treatment of lung neoplasms [5].

Also related to lung neoplasms, heme oxygenase-1 (HO-1) is the enzyme responsible for the breakdown of heme into biliverdin, ferrous iron and carbon monoxide (CO) [6]. Its expression increases in stressful conditions, becoming a protective factor against oxidative stress [6]. Besides, it acts on cell proliferation, modulating the inflammatory response and facilitating angiogenesis [6].

In neoplasms, high HO-1 expression is observed, aggravated by radiotherapy, chemotherapy, or photodynamic therapy [6]. Therefore, it is believed that $\mathrm{HO}-1$ is related to tumor induction and its evolution, as well as dissemination [6].

Additionally, in lung cancers, Ecto-5'Nucleotidase (CD73) is responsible for the conversion of extracellular adenosine monophosphate (AMP) into adenosine and inorganic phosphate [7]. The adenosine in this process binds to four adenosine receptors coupled to the $G$ protein, namely: $A 1, A 2 A, A 2 B$ and A3 [7]. In addition, in lung cancer, the A2A adenosine receptor is predominantly expressed [7]. Adenosine is responsible for inhibiting the performance of anti-tumor $\mathrm{T}$ cells, in addition to acting on the apoptosis of these same cells being directly related to immunosuppression [7]. Together, adenosine and CD73 trigger the proliferation of neoplastic cells, promote neovascularization and, consequently, enable the process of metastasis and chemo suppression of altered cells [7].

In addition, ectopurins and enzymes that participate in adenosine metabolism are present in the extracellular environment [8]. They are responsible for modulating nucleotide-related signaling [8]. Thus, molecules of ATP and adenosine diphosphate (ADP) are hydrolyzed into ecto nucleoside triphosphate diphosphohydrolase (E-NTPDases) and 5'nucleotidases in adenosine, which act by stimulating purinergic receptors and, consequently, inhibiting ATP degradation [8]. This review summarizes the performance of the purinergic system, especially the P2Y and P2X7 receptors, with regard to inhibition of cell proliferation [8]. 


\section{PURINERGIC SIGNALING}

Purinergic receptors are responsible for extracellular signaling, thus acting in several human processes, namely, endocrine and exocrine secretion, inflammatory process, pain mechanism, platelet aggregation, immune response and cell differentiation [9]. There are two types of purinergic receptors, classified as $\mathrm{P} 1$ and $\mathrm{P} 2$. The $\mathrm{P} 1$ receptor promotes cellular responses through the action of adenosine, whereas the P2 receptor, ATP [10]. P1 receptors are metabotropic receptors associated with protein $G$ and endogenously stimulated by the action of adenosine. When activated, these receptors modulate the intracellular concentrations of 3 ', 5'-monophosphate (cAMP) adenosine [10]. P2 receptors, however, have two subunits, P2X (ionotropic) and P2Y (metabotropic) [11]. P2X receptors are responsible for the formation of ion channels in the phospholipid bilayer of the cell membrane, which include sodium, potassium and calcium íons [11]. These receptors are subdivided into seven units (P2X1-7), each with two transmembrane alpha-propellers. $\mathrm{P} 2 \mathrm{X}$ receptors have an affinity for the ATP ligand or analogues [11].

P2Y receptors also group receptors that have uracil derivatives as agonists, characterized as pyrimidine receptors [10]. There are eight human $\mathrm{P} 2 \mathrm{Y}$ receptors, namely: P2Y1, P2Y2, P2Y4, P2Y6, P2Y11, P2Y12, P2Y13, P2Y14. P2Y receptors, as well as $\mathrm{P} 1$ receptors, are also associated with protein $\mathrm{G}$ [10] (Fig. 1).

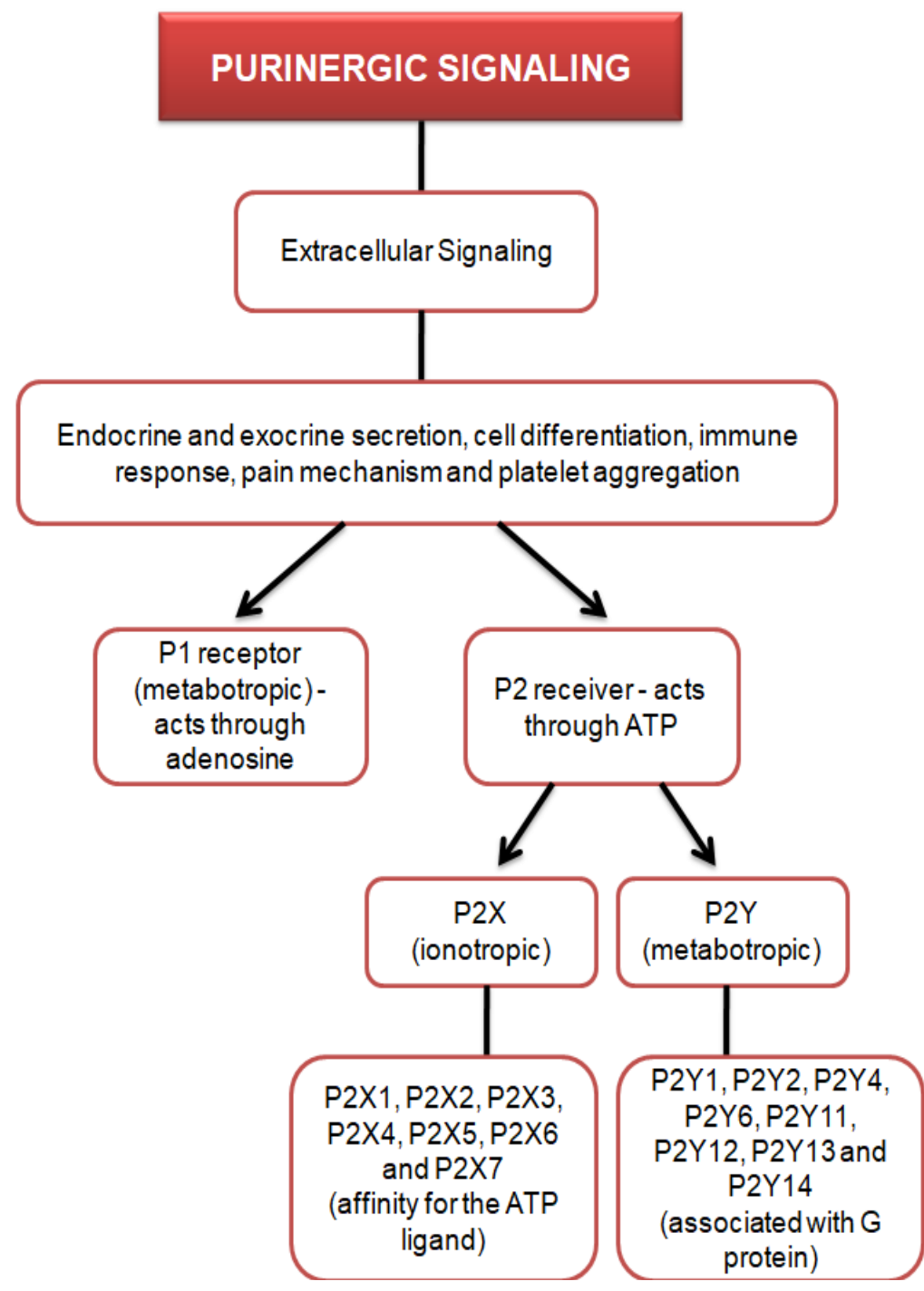

Fig. 1. Schematic representation of purinergic signaling Source: The authors, 2020 


\section{LUNG NEOPLASMS}

Lung neoplasms are characterized by cellular abnormalities of the organ, caused by the alteration of some genes, triggering an uncontrolled cell multiplication [12]. The habit of smoking predisposes to a process of chronic inflammation, responsible for inhibiting the cells of the immune system and, consequently, favoring the development of cancer cells [12]. In addition, the $\mathrm{P} 2 \mathrm{X} 7$ receptor plays an essential role in the inflammatory condition, since it activates the inflammatory NLRP3 and, consequently, the release of interleukin $\mathrm{IL}-1 \beta$ occurs, which has a pyrogenic function [12]. In more advanced stages of the disease, the detection of neoplasms usually occurs in stage IV, when a process of metastasis to lymph and blood vessels occurs [12]. It is also noticed that lung carcinomas have some preferred sites of metastasis, such as the brain, bones and adrenal glands [12].

The risk of cancer decreases considerably in exsmokers, but the chances of disease progression remain great even after years without smoking [12]. However, non-smokers exposed to cigarette smoke daily are likely to contract the disease, which is the seventh most common cause of death among them [12]. Another point of concern is the environmental factors involved in lung cancer, including, in addition to active and passive smoking, air pollution, exposure to carcinogenic chemicals and alcohol consumption [12]. In addition, exposure to radon, heavy metals and asbestos, significantly increases the risk of developing cancer [13,14].

Furthermore, genetic predisposition is a fundamental aspect, since even those who have never had the habit of smoking, but have family members with a history of lung cancer, are susceptible to this [15]. Few genes have been associated with a high risk of lung cancer, but some pathogenic variants in the TP53 gene have been linked to early disease onset [15]. In genetic epidemiology, recent studies have pointed out RGS17 as a possible cancer susceptibility gene, however, it still needs to be replicated and its functional impact remains uncertain [15].

Lung cancer has a complex therapeutic resource that requires the integrated participation of a multidisciplinary team. For the treatment to occur properly, a biopsy must be performed, followed by staging tests and, thus, it is possible to determine the stage of the disease. Therefore, in patients who present the pathology without metastases, the surgical procedure is performed, with or without chemotherapy and radiotherapy sessions [16]. On the other hand, when the disease is located in the lungs and also in the lymph nodes, the method used is to carry out chemotherapy and radiotherapy sessions simultaneously [16]. In the case of patients with metastases, chemotherapy sessions are performed and, in some cases, therapy with drugs that stabilize neoplastic growth is used as a target [16].

There are two main subtypes of lung cancer, small cell lung cancer and non-small cell lung cancer, which are classified as: squamous cell carcinoma, adenocarcinoma and large cell carcinoma [17]. Among these, adenocarcinoma, which represents $40 \%$ of lung cancers, is derived from epithelial cells that line the secretory glands [17]. In addition, compared to other types of lung cancer, adenocarcinoma tends to grow more slowly and is more likely to be found before metastasizing and causing more serious complications [17]. It tends to occur at the periphery of the lung and, depending on its location, it may take time to show symptoms. However, it usually causes bloody cough, dyspnea, chest pain and weight loss [17].

Squamous cell carcinoma comprises 25 to $30 \%$ of all cases of lung câncer [17]. It is initially manifested in the airway epithelial cells in the bronchial tubes in the center of the lungs [17]. Large cell carcinoma represents a minority of small cell cancer cases, with approximately $3 \%$ of small cell cancer cases. They are usually located peripherally, bulky and necrotic in appearance [18].

\section{P2Y RECEIVER}

Increased levels of ATP have been found in lung cancer 100 to 200 times. These molecules act on $\mathrm{P} 2$ receptors, which can be ligand-dependent ion channels (P2X) or muscarinic receptors (P2Y) [19].

In pulmonary adenocarcinomas, ATP acts by stimulating cell proliferation by binding to the P2Y receptor. This causes calcium levels to increase, interfering with cell survival and death [19]. Cell proliferation related to $\mathrm{P} 2 \mathrm{Y}$ activation depends on the transcription factor NF-KB, which also acts on the transcription of the Bax gene (related to the apoptosis process) [19]. 
The Bax gene thus allows the opening of the mitochondrial ion channel, releasing cytochrome $\mathrm{C}$ and pro-apoptotic factors [19]. This promotes the release of caspases. However, in lung cancer, the action of the Bax gene can also be anti-apoptotic, promoting the survival of these cells [19].

\section{P2X7 RECEIVER}

The P2X7 receptor is present in many malignancies, including lung cancers [19]. This has a significant role in cellular responses, which, after deregulation, are associated with tumor initiation and development [19]. The action of ATP provides a flow of cations in the cell. However, prolonged activation of P2X7 causes the formation of pores, which increases cell permeability and eventually promotes cell death. This fact is a paradox, considering that at times the recipient promotes greater survival [19].

Exposure to high concentrations of ATP, present in tumor environments, leads to the expression of NfP2X7, essential for the survival of neoplastic cells [19]. Also, the data indicate the relevance of the P2X7 receptor for tumor growth, which was verified by the depletion of the study receptor. From this, there is an acceleration of apoptosis [19].

In this context, a relevant lung cancer subtype is malignant human pleural mesothelioma, of mesothelial origin [20]. In the cancer cells of mesothelioma, there is the presence of P2X7 receptors, which are not found in healthy cells. It is suggested, therefore, that this receptor is related to cell proliferation, as already mentioned [20]. However, specifically in malignant human pleural mesothelioma, there are still uncertainties about the involvement of the purinergic system in tumor progression [20].

In the research carried out, it was observed that the proliferation of malignant cells of human pleural mesothelioma was inhibited by in vitro incubation in the presence of selective P2X7 receptor antagonists. This corroborates the theory that the P2X7 receptor is related to the proliferation of malignant cells [20].

\section{SUPPRESSION OF P2Y RECEPTORS AND INHIBITION OF CELL PROLIFERATION}

In neoplastic cells, the concentration of ATP is higher compared to non-neoplastic cells [19]. Thus, the ATP concentration is responsible for regulating P2Y receptors, triggering their activation, as well as providing cell multiplication [19]. For the activation of P2Y receptors, it is necessary to activate the NF-kB gene [19]. Therefore, the inhibition of this signaling pathway reduces cell proliferation, especially in pulmonary adenocarcinomas, since there is a modulation of calcium levels induced by ATP and, consequently, the inhibition of cell cycle progression [19].

\section{ANTINEOPLASTIC MECHANISMS}

Studies indicate that the ZnLos complex (zinc and losartan) in concentrations up to $500 \mu \mathrm{m}$, acts in the promotion of reactive oxygen species and, consequently, in cell cytotoxicity, triggering the apoptosis of neoplastic cells [21]. Furthermore, this complex regulates BAX protein positively and bcl-XL protein negatively, in addition to acting on caspase-3 release, allowing the greater performance of apoptotic cells [21]. Additionally, metformin, a drug used to treat type 2 diabetics, works by slowing the progression of non-small cell lung cancers by inhibiting the expression of heme oxygenase 1 (HO-1) [22]. Metformin also increases the generation of reactive oxygen species, which is induced by epigallocatechin-3-gallate (EGCG) $(100 \mu \mathrm{m})$ and results in apoptosis [22]. Thus, it appears that HO-1 promotes greater resistance to chemotherapeutic drugs such as EGCG [22].

E-cadherin is a surface protein responsible for intercellular adhesion, which determines cell polarity and differentiation [23]. Its decrease in tumor processes promotes increased angiogenesis, as well as adherence disorder, which modifies the signaling processes and, therefore, promotes the progression of the neoplasm. It has been observed that this protein is altered in non-small cell lung cancers [23].

\section{CONCLUSION}

In conclusion, the P2Y and P2X7 purinergic receptors are directly related to lung cancer, so that they act by inhibiting the proliferation of neoplastic cells $[23,24]$. Thus, it is observed that clinical research correlating purinergic receptors with the treatment of lung cancer needs to be explored in order to bring to the scientific community a better understanding of the performance of these receptors, specifically $\mathrm{P} 2 \mathrm{X} 7$ and P2Y, as well as their importance as targets for drugs antineoplastic [22]. Moreover, it was observed that E-cadherin is involved in the process of tumor progression and the drugs 
related to it are of clinical importance and should be better explored [23].

\section{FUTURE PERSPECTIVES}

From studies already carried out, it is known that high concentrations of ATP act to stimulate the progression of neoplastic cells, triggering metástases [25]. Extracellular ATP increases the intracellular ATP concentration and therefore promotes cell growth and survival [25]. Thus, it is expected that drugs can be developed to effiently inhibit intracellular calcium concentrations in the control of ATP-induced neoplastic growth. In addition, the discovery of a selective inhibitor of the P2Y receptor, to regulate calcium concentrations, would be beneficial in terms of controlling the process of tumorigenesis [25]. Besides, the inhibition of the activation of the calcium-dependent NF-kB signaling pathway is necessary for cell control. Furthermore, it is expected that, with scientific progress, specific control mechanisms of the Bcl-2 protein family can be explored to regulate the activation or inhibition of anti-apoptotic or pro-apoptotic functions, so that the cells responsible for neoplastic development are eliminated, avoiding possible metástases [25].

In addition to the P2Y purinergic receptor, the P2X7 receptor is directly related to the process of lung tumorigenesis. The reduction of the levels of the P2X7 protein would provide the reduction of uncontrolled cell migration induced by ATP [26]. The great challenge is to carry out this cellular control without causing serious damage to human beings, both at the micro and macroscopic levels, considering that every scientific advance must be supported by respect and the dignity of human life [26]. Also, it is expected that, over time, access to information will be more widespread among the world population, so that harmful habits such as smoking can be internalized as something destructive to human life, since many comorbidities are associated with smoking, including lung cancer.

\section{COMPETING INTERESTS}

Authors have declared that no competing interests exist.

\section{REFERENCES}

1. Torre LA, Bray F, Siegel RL, Ferlay J, Lortet-Tieulent J, Jemal A. Global cancer statistics, 2012. CA Cancer J Clin. 2015; 65:87-108.

2. Kim $\mathrm{CH}$, Lee $\mathrm{YC}$, Hung RJ, Mc Nallan SR, Cote ML, Lim WY et al. Exposure to secondhand tobacco smoke and lung cancer by histological type: A pooled analysis of the International Lung Cancer Consortium (ILCCO). Int J Cancer. 2014; 135:1918-30.

3. Lin WW, Karin M. A cytokine-mediated link between innate immunity, inflammation, and cancer. J Clin Invest. 2007;117:117583.

4. Ma J, Li W, Chai Q, Tan X, Zhang K. Correlation of P2RX7 gene rs1718125 polymorphism with postoperative fentanyl analgesia in patients with lung cancer. Medicine (Baltimore). 2019;98:e14445.

5. Miras-Portugal MT, Queipo MJ, GilRedondo JC, Ortega F, Gómez-Villafuertes $\mathrm{R}$, Gualix J et al. P2 receptor interaction and signalling cascades in neuroprotection. Brain Res Bull. 2018;151: 74-83.

6. Was H, Dulak J, Jozkowicz A. Heme oxygenase-1 in tumor biology and therapy. Curr Drug Targets. 2010;11:1551-70.

7. Jin D, Fan J, Wang L, Thompson LF, Liu A, Daniel BJ et al. CD73 on tumor cells impairs antitumor T-cell responses: $\mathrm{A}$ novel mechanism of tumor-induced immune suppression. Cancer Res. 2010; 70:2245-55.

8. Burnstock G, Di Virgilio F. Purinergic signalling and cancer. Purinergic Signal. 2013;9:491-540.

9. North RA. Molecular physiology of P2X receptors. Physiol Rev. 2002;82:1013-67.

10. Turner CM, Elliott JI, Tam FW. P2 receptors in renal pathophysiology. Purinergic Signal. 2009;5:513-20.

11. Burnstock G. Purine and pyrimidine receptors. Cell Mol Life Sci. 2007;64:147183.

12. Popper HH. Progression and metastasis of lung cancer. Cancer Metastasis Rev. 2016;35:75-91.

13. Kanwal M, Ding XJ, Cao Y. Familial risk for lung cancer. Oncol Lett. 2017;13:535-42.

14. Stefanescu SV, Buciu A, Al-Saffar H, Cirin $A B$. Dental perspective on radioactive nuclides in humans living on the premises of uranium mining facilities. J Dent Maxillofacial Res. 2020;3:1-5.

15. Koeller DR, Chen R, Oxnard GR. Hereditary lung cancer risk: Recent discoveries and implications for genetic 
counseling and testing. Current Genetic Medicine Reports. 2018;6:83-88.

16. Denton E, Conron M. Improving outcomes in lung cancer: The value of the multidisciplinary health care team. J Multidiscip Healthc. 2016;9:137-44.

17. Zappa C, Mousa SA. Non-small cell lung cancer: Current treatment and future advances. Transl Lung Cancer Res. 2016; 5:288-300.

18. Zheng M. Classification and pathology of lung cancer. Surg Oncol Clin. 2019;8:447468 .

19. Wang X, Li L, Guan R, Zhu D, Song N, Shen L. Emodin inhibits ATP-induced proliferation and migration by suppressing P2Y receptors in human lung adenocarcinoma cells. Cell Physiol Biochem. 2017;44:1337-51.

20. Gilbert SM, Oliphant CJ, Hassan S, Peille AL, Bronsert $\mathrm{P}$, Falzoni $\mathrm{S}$ et al. ATP in the tumour microenvironment drives expression of nfP2X. Oncogene. 2019;38: 194-208.

21. Martínez VR, Aguirre MV, Todaro JS, Piro OE, Echeverría GA, Naso LG et al. Interaction of $\mathrm{Zn}$ with losartan. Activation of intrinsic apoptotic signaling pathway in lung cancer cells and effects on alkaline and acid phosphatases. Biol Trace Elem Res. 2018;186:413-29.

22. Yu C, Jiao $Y$, Xue J, Zhang $Q$, Yang $H$, Xing $L$ et al. Metformin sensitizes nonsmall cell lung cancer cells to an epigallocatechin-3-gallate (EGCG) treatment by suppressing the $\mathrm{Nrf} / \mathrm{HO}-1$ signaling pathway. Int J Biol Sci. 2017; 13:1560-9.

23. Lin $\mathrm{CC}$, Lu CH, Pan $\mathrm{YH}$, Jiao L, Chen HY, $\mathrm{Li} L$ et al. Effect and mechanism of silibinin on the inhibition of ALK positive NSCLC cells by sensitizing crizotinib. Zhonghua Zhong Liu Za Zhi. 2017;39:650-6.

24. Campos-Contreras AR, Díaz-Muñoz M, Vázquez-Cuevas FG. Purinergic signaling in the hallmarks of cancer. Cells. 2020;9:124.

25. Song $S$, Jacobson $K N$, McDermott $K M$, Reddy SP, Cress AE, Tang $\mathrm{H}$ et al. ATP promotes cell survival via regulation of cytosolic [Ca2+] and $\mathrm{Bcl}-2 / \mathrm{Bax}$ ratio in lung cancer cells. Cell Physiology. 2016;310:99114.

26. Lara R, Adinolf E, Harwood CA, Philpott M, Barden JA, Di Virgilio $F$ et al. P2X7 in cancer: From molecular mechanisms to therapeutics. Front Pharmacol. 2020;11: 793.

(c) 2020 Bizuti et al.; This is an Open Access article distributed under the terms of the Creative Commons Attribution License (http://creativecommons.org/licenses/by/4.0), which permits unrestricted use, distribution, and reproduction in any medium, provided the original work is properly cited. 